\title{
Protein palmitoylation is critical for the polar growth of root hairs in Arabidopsis
}

\author{
Yu-Ling Zhang, En Li, Qiang-Nan Feng, Xin-Ying Zhao, Fu-Rong Ge, Yan Zhang and Sha Li
}

\begin{abstract}
Background: Protein palmitoylation, which is critical for membrane association and subcellular targeting of many signaling proteins, is catalyzed mainly by protein S-acyl transferases (PATs). Only a few plant proteins have been experimentally verified to be subject to palmitoylation, such as ROP GTPases, calcineurin B like proteins (CBLs), and subunits of heterotrimeric $G$ proteins. However, emerging evidence from palmitoyl proteomics hinted that protein palmitoylation as a post-translational modification might be widespread. Nonetheless, due to the large number of genes encoding PATs and the lack of consensus motifs for palmitoylation, progress on the roles of protein palmitoylation in plants has been slow.

Results: We combined pharmacological and genetic approaches to examine the role of protein palmitoylation in root hair growth. Multiple PATs from different endomembrane compartments may participate in root hair growth, among which the Golgi-localized PAT24/TIP GROWTH DEFECTIVE1 (TIP1) plays a major role while the tonoplast-localized PAT10 plays a secondary role in root hair growth. A specific inhibitor for protein palmitoylation, 2-bromopalmitate (2-BP), compromised root hair elongation and polarity. Using various probes specific for cellular processes, we demonstrated that 2-BP impaired the dynamic polymerization of actin microfilaments (MF), the asymmetric plasma membrane (PM) localization of phosphatidylinositol (4,5)-bisphosphate ( $\left.\mathrm{PIP}_{2}\right)$, the dynamic distribution of RabA4b-positive post-Golgi secretion, and endocytic trafficking in root hairs.

Conclusions: By combining pharmacological and genetic approaches and using root hairs as a model, we show that protein palmitoylation, regulated by protein S-acyl transferases at different endomembrane compartments such as the Golgi and the vacuole, is critical for the polar growth of root hairs in Arabidopsis. Inhibition of protein palmitoylation by 2-BP disturbed key intracellular activities in root hairs. Although some of these effects are likely indirect, the cytological data reported here will contribute to a deep understanding of protein palmitoylation during tip growth in plants.
\end{abstract}

Keywords: Polar growth, 2-bromopalmitate, TIP1, Actin microfilaments, Endocytosis

\section{Background}

Protein palmitoylation, or S-acylation, is a reversible post-translational modification that adds a 16-carbon saturated palmitate group to the sulfhydryl group of a cysteine to form a thioester [1-3]. Such modifications affect protein trafficking, protein interactomes and protein stability [1-3]. Palmitoylation, usually combined with other lipid modifications such as N-myristolyation and prenylation, provides a hydrophobic membrane anchor on otherwise soluble proteins, enhancing their membrane association $[1,2,4]$. Transmembrane (TM)

\footnotetext{
*Correspondence: shali@sdau.edu.cn

State Key Laboratory of Crop Biology, College of Life Sciences, Shandong Agricultural University, Tai'an 271018, China
}

proteins, such as receptor kinases and transporters, can also be modified by palmitoylation, which often affects their subcellular targeting and dynamic sorting among different endomembrane compartments [1].

Palmitoyl proteomics indicated that eukaryotes contain a large number of palmitoylated proteins [1,5-7]. Most of palmitoylated proteins, such as small GTPases, receptor tyrosine kinases, transporters, and $\mathrm{N}$-ethylmaleimide-sensitive factor-activating protein receptors (SNAREs), are involved in cell signaling and intracellular transport [1,5-7]. In plants, a few proteins have been experimentally demonstrated to be modified by palmitoylation, including ROP GTPases [8-10], CBLs [11,12], subunits of heterotrimeric G proteins [13,14], protein phosphatases [15], and the 
receptor kinase FLAGELLIN-SENSING 2 [5]. Modification of these key signaling proteins implies that palmitoylation plays crucial roles in plant growth.

Three types of enzymes are reported to catalyze protein palmitoylation [3], among which protein S-acyl transferases (PATs), characterized by an evolutionarily conserved and catalytically critical Asp-His-His-Cys (DHHC) motif within a cysteine-rich domain, play dominant roles $[1,3]$. DHHC-type PATs are encoded in all eukaryotic genomes [1]. As transmembrane ${ }^{\text {Tn }}$ proteins, PATs are found at different endomembrane compartments including the Golgi, endoplasmic reticulum (ER), the plasma membrane (PM), and vacuolar membrane in yeast [16]. Recently, it was shown that Arabidopsis PATs have more diverse targeting than their yeast or metazoan counterparts, at the PM, the Golgi, ER, the tonoplast, or various vesicles of distinct identities [17]. Two plant PATs have been functionally characterized [12,18]. Arabidopsis TIP GROWTH DEFECTIVE1 (TIP1)/PAT24 encodes a PAT with ankyrin repeats, whose mutations result in defective growth both in tip-growing cells, i.e. root hairs and pollen tubes, and in non-tip-growing cells [18-20]. We recently characterized a tonoplast-localized PAT, PROTEIN S-ACYL TRANSFERASE10 (PAT10), which is critical for vacuolar function [12]. In the pat10 mutants, several CBLs lost their palmitoylation-dependent tonoplast association [12], suggesting that these CBLs are the substrates of PAT10.

Despite the importance of protein palmitoylation for plant growth, progress in understanding plant PAT functions has been slow due to redundancy and overlapping substrate specificity $[1,2]$. We report here that protein palmitoylation regulates the polar growth of root hairs by using a pharmacological approach in combination with genetics. Root hair growth requires the dynamic distribution of intracellular activities such as actin MF [21-24] and membrane trafficking [24-30]. Many proteins mediating such dynamic activities are likely regulated by palmitoylation based on evolutionary conservation [1] or results from palmitoyl proteomics [5]. Thus, root hairs represent an excellent single cell system to study the effect of protein palmitoylation on multiple intracellular activities.

We show that the Golgi-localized TIP1 plays a major role while the tonoplast-localized PAT10 plays a minor role in the polar growth of root hairs. By application of 2-bromopalmitate (2-BP) that specifically inhibits protein palmitoylation in vitro [31] and in planta [11,12,18], we show here that inhibiting palmitoylation directly or indirectly impaired actin MF polymerization, abolished the restricted $\mathrm{PM}$ localization of $\mathrm{PIP}_{2}$, disrupted the dynamic distribution of RabA4b-positive post-Golgi secretion, and inhibited vacuolar trafficking, resulting in defective root hair growth. Thus our results demonstrate the role of protein palmitoylation in intracellular activities that contribute to cell morphogenesis in root hairs and provide experimental evidences to narrow down potential PAT targets in plants.

\section{Results}

Optimization of 2-BP treatment on root hair growth

To examine cellular processes affected by 2-BP in root hairs, it was necessary to develop a suitable treatment regime that would reveal the effects of inhibiting protein palmitoylation on cellular processes without causing severe cellular damages. To do so, we utilized the subcellular localization of CBL2 as an indicator for the effective inhibition of palmitoylation. CBL2 dissociated from the tonoplast and moved to the cytoplasm when its key palmitoylation site was mutated [11] or in the pat10 mutants [12]. Based on previous studies [11,12,18], we added 2-BP at a final concentration of $10 \mu \mathrm{M}$ to $50 \mu \mathrm{M}$ to Pro $_{U B Q 10}$ :CBL2-RFP transgenic seedlings 4 days after germination (DAG) in a hypotonic MS solution, to determine the effects on the tonoplast association of CBL2. Because 2-BP was dissolved in dimethyl sulfoxide (DMSO), equivalent volumes of DMSO were applied as controls in which no phenotypic consequences were detected over the time course of the experiments (Figure 1A,B). As an additional control, we also introduced the same Pro ${ }_{U B Q 10}:$ CBL2-RFP transgene into pat10-2 by crosses [12], in which CBL2 was rendered cytosolic (Figure 1E,F). As expected, 2-BP treatment resulted in gradual relocalization of CBL2 from the tonoplast to the cytoplasm of root hairs (Figure 1). Root hairs incubated with $10 \mu \mathrm{M} 2-\mathrm{BP}$ for $2-3 \mathrm{hrs}$ showed the most substantial reduction of CBL2 at the tonoplast (Figure 1G) and at $12 \mathrm{hrs}$ showed complete absence of tonoplast-CBL2 (Figure 1). Increasing 2-BP concentration from $10 \mu \mathrm{M}$ to $50 \mu \mathrm{M}$ did not substantially accelerate the tonoplast dissociation of CBL2 but induced extensive vacuolation (Additional file 1: Figure S1). Based on these results, we used $10 \mu \mathrm{M} 2$-BP on root hairs and examined cellular processes from 2 to $12 \mathrm{hrs}$ after 2-BP application for further experiments.

\section{Root hair growth was impaired by 2-BP}

We examined the effect of inhibiting protein palmitoylation on the initiation and polar growth of root hairs. Application of $10 \mu \mathrm{M} 2$-BP for $12 \mathrm{hrs}$ did not cause a substantial change in primary root length (Figure 2A). However, compared to roots treated with DMSO, those treated with 2-BP showed a much expanded region of root hair initiation (Figure 2A), suggesting inhibited root hair elongation. Indeed, the polar growth of root hairs was significantly affected by 2 -BP treatment, such that root hairs were shorter and wider than those treated with DMSO at the maturation zone (Figure 2D,E,F,G). In addition, 2-BP caused extensive vacuolation in growing 


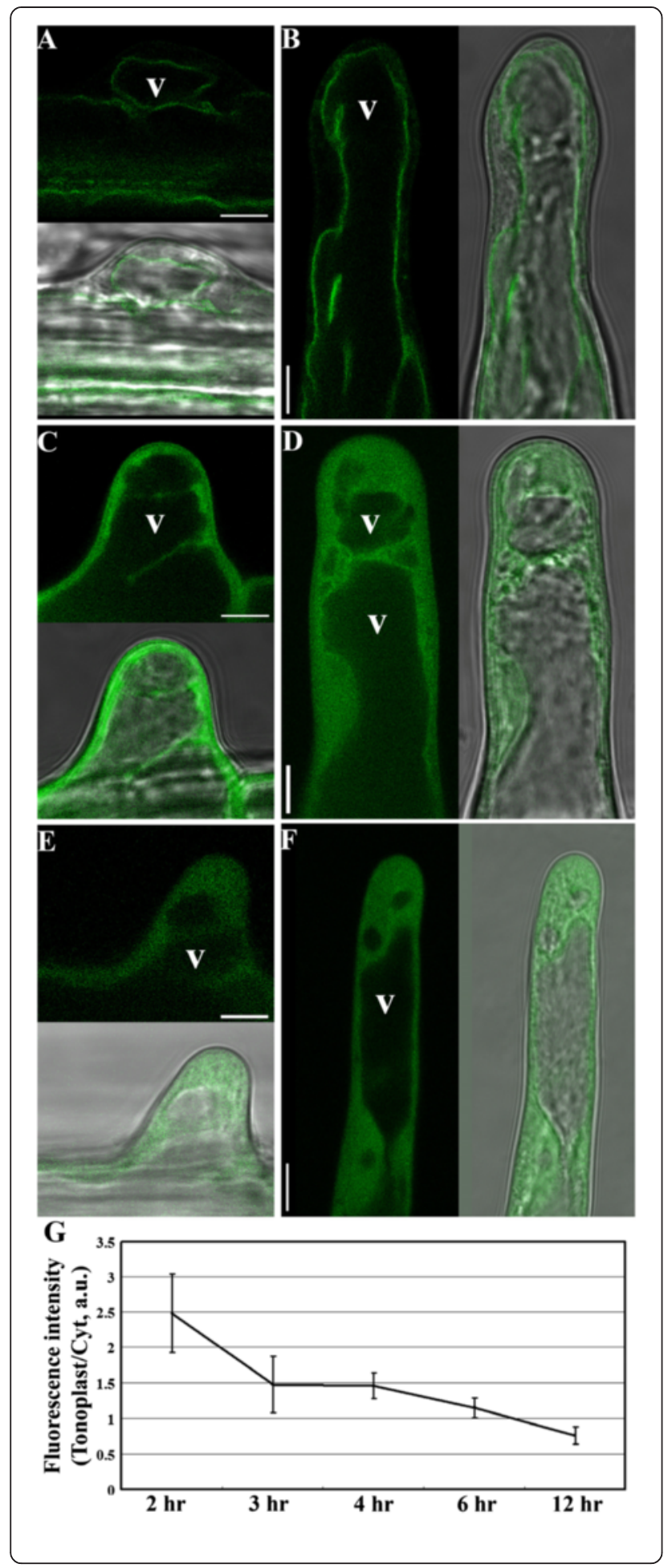

Figure $12-\mathrm{BP}$ abolished the tonoplast localization of $\mathrm{CBL} 2$ in root hairs. A-D. 4 DAG seedlings of Pro ${ }_{U B Q} 10: C B L 2-R F P$ transgenic plants treated with either DMSO (A, B) or 2-BP (C, D) for 4-12 hr before visualization. E-F. 4 DAG seedlings of ProuBQ10:CBL2-RFP;pat102 transgenic plants treated with DMSO for 4-12 hr before imaging. Representative initiating $(A, C, E)$ or elongating $(B, D, F)$ root hairs are shown. V indicates vacuole. G. Quantification of CBL2-RFP distribution in the tonoplast v.s. the cytoplasm (Tonoplast/Cyt) at different time points after 2-BP treatment. a.u. stands for arbitrary fluorescence units. Bars $=7.5 \mu \mathrm{m}$.

root hairs, compared to root hairs treated only with DMSO (Figure 2D,E).

Most Arabidopsis PATs represented on the ATH1 Chip [32] are expressed in root hairs or pollen tubes (Additional file 1: Figure S2). Indeed, tip1 mutants exhibited defective growth in both root hairs and pollen tubes [18-20]. TIP1 was shown to be Golgi-localized [17] by using transient expression in tobacco epidermal cells. However, transient heterogeneous expression with strong constitutive promoters does not always reflect the native localization of proteins, as is the case for PAT10 $[12,17]$. To determine its native localization, we introduced a TIP1 genomic fragment-GFP translational fusion driven by its native promoter (TIP1g-GFP) into tip1-4, a novel null mutant (Additional file 1: Figure S3). TIP1gGFP fully restored the root hair defects of tip1-4 (Figure 3D,E,F), indicating that the GFP fusion did not interfere with its functionality. To verify that the punctate vesicles labeled by TIP1 were of Golgi identity, we applied the lipophilic dye FM4-64 and the fungal toxin Brefeldin A (BFA) to TIP1g-GFP;tip1-4 roots. FM4-64 enters cells via endocytic trafficking and sequentially labels trans-Golgi network/early endosomes (TGN/EE), prevacuolar compartment/multivesicular bodies (PVC/ $\mathrm{MVB})$, then finally reaches the tonoplast [33]. BFA interferes with the activity of Arf GTPases and its application resulted in the formation of so-called BFA compartments with a TGN/EE core surrounded by aggregates of Golgi [34]. FM4-64 uptake together with BFA treatment confirmed the localization of TIP1 at the Golgi (Additional file 1: Figure S4). To find out whether TIP1 played a dominant role in root hair growth, we applied 2-BP to tip1-4 roots and analyzed root hair morphology. There were slight but not significant morphological changes to root hair length and width in 2-BP-treated tip1-4 (Figure 2C,D,E,F), suggesting that TIP1 is a major PAT functioning in root hairs.

PAT10 is also expressed in root hairs (Additional file 1: Figure S2). However, it was unclear whether PAT10 played a role in root hair growth [12]. In PAT10g-GFP; pat10-2, PAT10 was localized at the tonoplast of root hairs at all stages (Figure $3 \mathrm{~A}, \mathrm{~B}, \mathrm{C}$ ). We therefore analyzed the root hair morphology of pat10-2 in the absence or presence of 2-BP. Root hair initiation and 


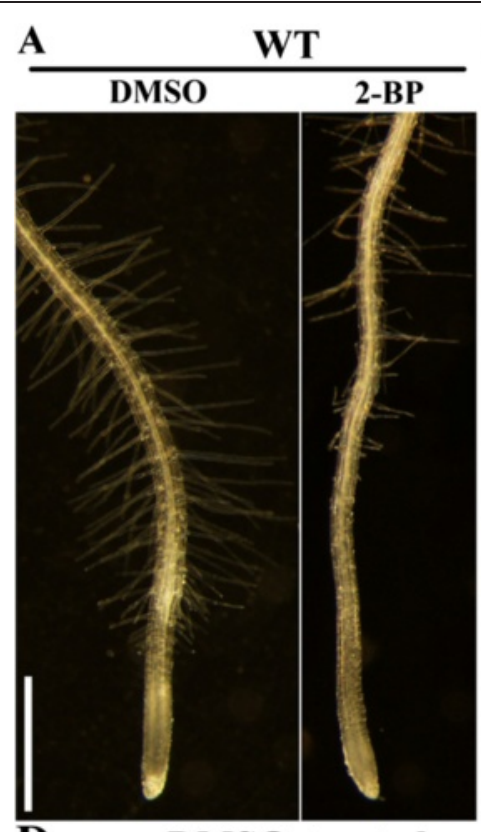

\section{B

B pat10-2 \\ $\frac{\text { C tip 1-4 }}{\text { DMSO 2-BP }}$}
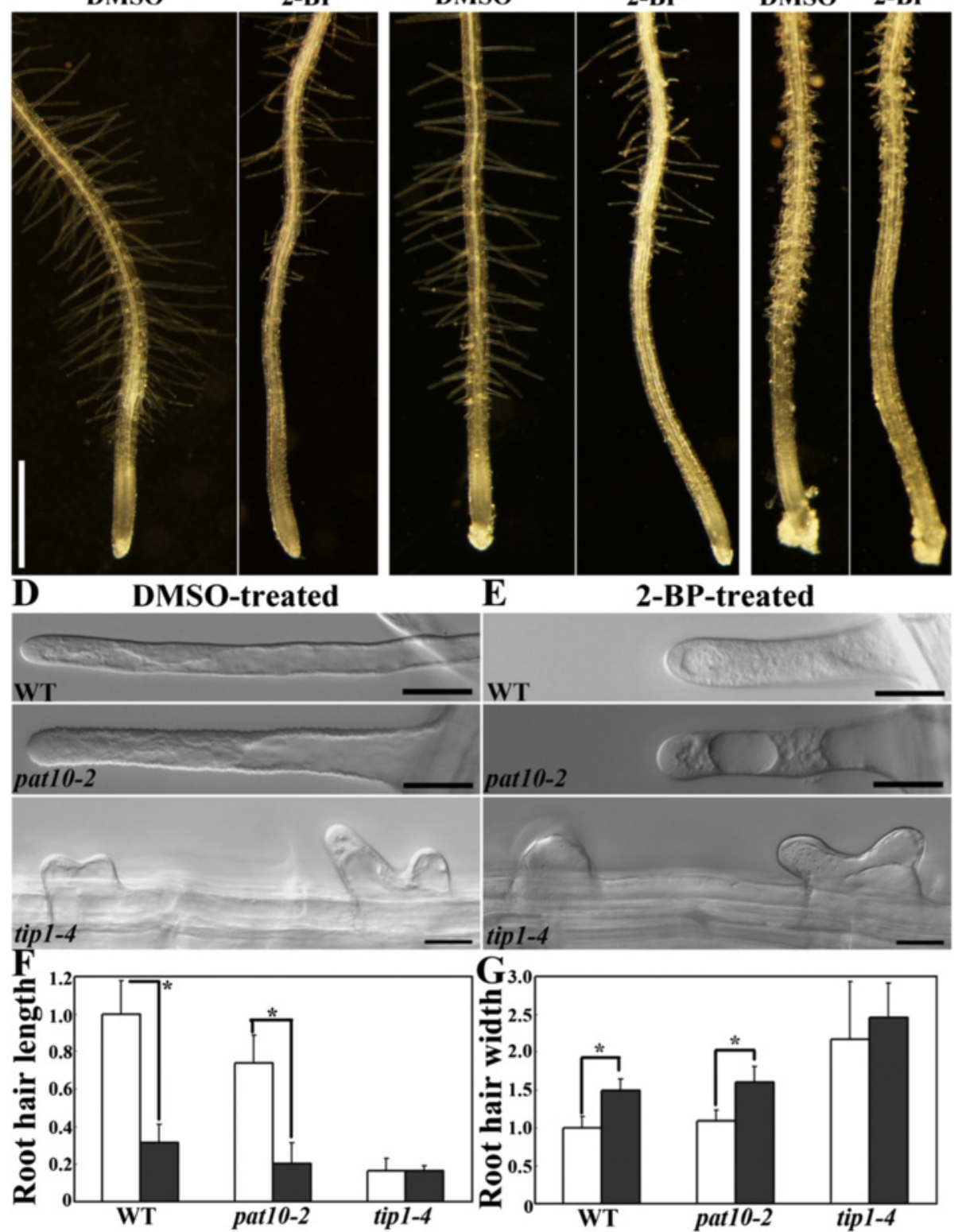

Figure 2 2-BP impaired root hair growth. A. Primary roots from 4 DAG seedlings of wild type treated with either DMSO or 2-BP. B. Primary roots from 4 DAG seedlings of pat10-2 treated with either DMSO or 2-BP. C. Primary roots from 4 DAG seedlings of tip 1-4 treated with either DMSO or 2-BP. D-E. Representative root hairs at the hair elongation zone of 4 DAG seedlings treated with either DMSO (D) or with 2-BP (E). F-G. Root hair length (F) and width (G). Results are means \pm standard errors (SE), $N=4$. Length or width of mature wild-type root hairs treated with DMSO was set as 1. Empty bars represent DMSO treatment while filled bars represent 2-BP treatment. Asterisks indicate significant difference (Student's $t$-test, $P<0.05)$. Bars $=500 \mu \mathrm{m}$ for $(\mathbf{A}-\mathbf{C}) ; 20 \mu \mathrm{m}$ for $(\mathbf{D}-\mathbf{E})$.

polarity was not affected, as hair width was comparable between WT and pat10-2 (Figure 2G). However, root hair length was significantly reduced by PAT10 loss-offunction (Figure 2F). Treatment of 2-BP resulted in similar defects in pat10-2 as in wild type (Figure 2A), i.e. root hair initiation was substantially inhibited (Figure 2B). These results suggest that protein palmitoylation is important for root hair growth, with TIP1 plays a major role and other PATs, such as PAT10, also participating.

\section{2-BP disrupts actin MF polymerization and the} asymmetric PM localization of $\mathrm{PIP}_{2}$

Because 2-BP treatment significantly affected the polar growth of root hairs, we explored the underlying 


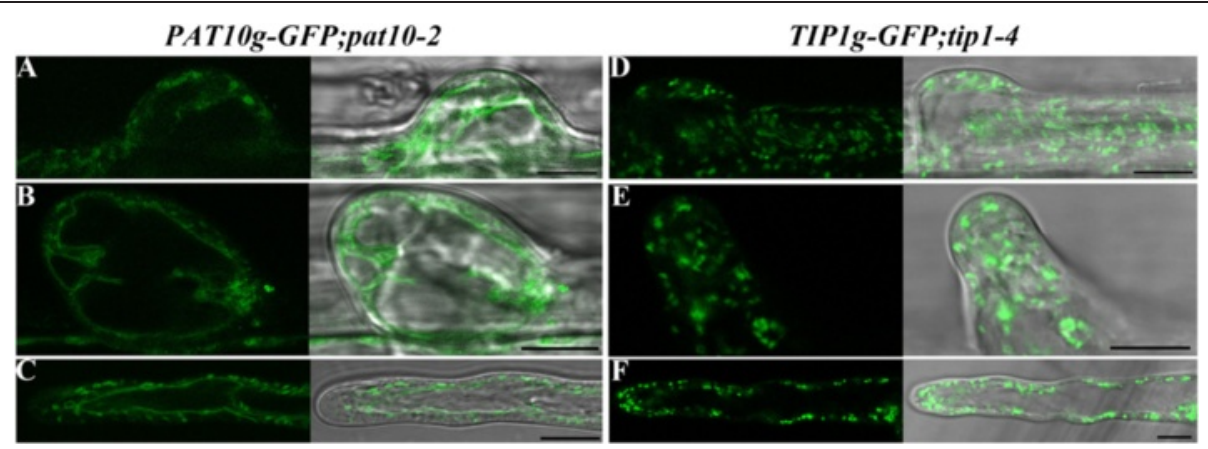

Figure 3 TIP1 and PAT10 localize at different endomembrane compartments in root hairs. A-C. Representative initiating root hair (A), elongating root hair (B), or mature root hair (C) of 4 DAG PAT10g-GFP;pat10-2 transgenic seedlings. D-F. Representative initiating root hair (D), elongating root hair (E), or mature root hair (F) of 4 DAG TIP1g-GFP;tip 1-4 transgenic seedlings. Bars $=10 \mu \mathrm{m}$.

mechanisms by examining the effects of 2-BP on critical intracellular activities during root hair growth such as the dynamic polymerization of actin MF [21-24] and the asymmetric PM distribution of $\mathrm{PIP}_{2}$ [35-37]. To analyze actin MF dynamics, we treated Arabidopsis transgenic plants expressing GFP-ABD2-GFP, which specifically labels actin MF [24,38-40] with either $10 \mu \mathrm{M} 2-\mathrm{BP}$ or DMSO and examined the pattern of actin MF in root hairs. In root hairs treated with DMSO, longitudinal or slightly helical actin cables extended to the subapical region from the base while short actin bundles as indicated by punctate filamentous signals were present in the apical region where active growth occurred (Figure 4). By contrast, treatment with 2-BP caused fragmentation as well as extensive cross-linking of actin MF (Figure 4). As a result, few longitudinal actin cables were observed in 2-BP-treated bulging root hairs (Figure 4).
Instead, numerous short actin filaments formed a meshlike network extending to the apical region (Figure 4). In elongating root hairs under 2-BP treatment, actin cables along the root hair shank were dotted with punctate actin aggregates (Figure 4). These effects occurred as quickly as 2-3 hrs after 2-BP treatment, indicating the sensitivity of the dynamic polymerization of actin MF. Treatment of root hairs with the actin MF depolymerization drug Latrunculin B (LatB) indicated that depolymerization of actin MF did result in punctate aggregates (Additional file 1: Figure S5). However, LatB treatment did not cause extensive cross-linking of actin MF in root hairs (Additional file 1: Figure S5), in contrast to those treated with 2-BP (Figure 4). These results suggest that the effect of 2-BP on actin MF polymerization is complex.

To determine the effect of 2-BP on the asymmetric $\mathrm{PM}$ localization of $\mathrm{PIP}_{2}$, we treated a P15Y fluorescence

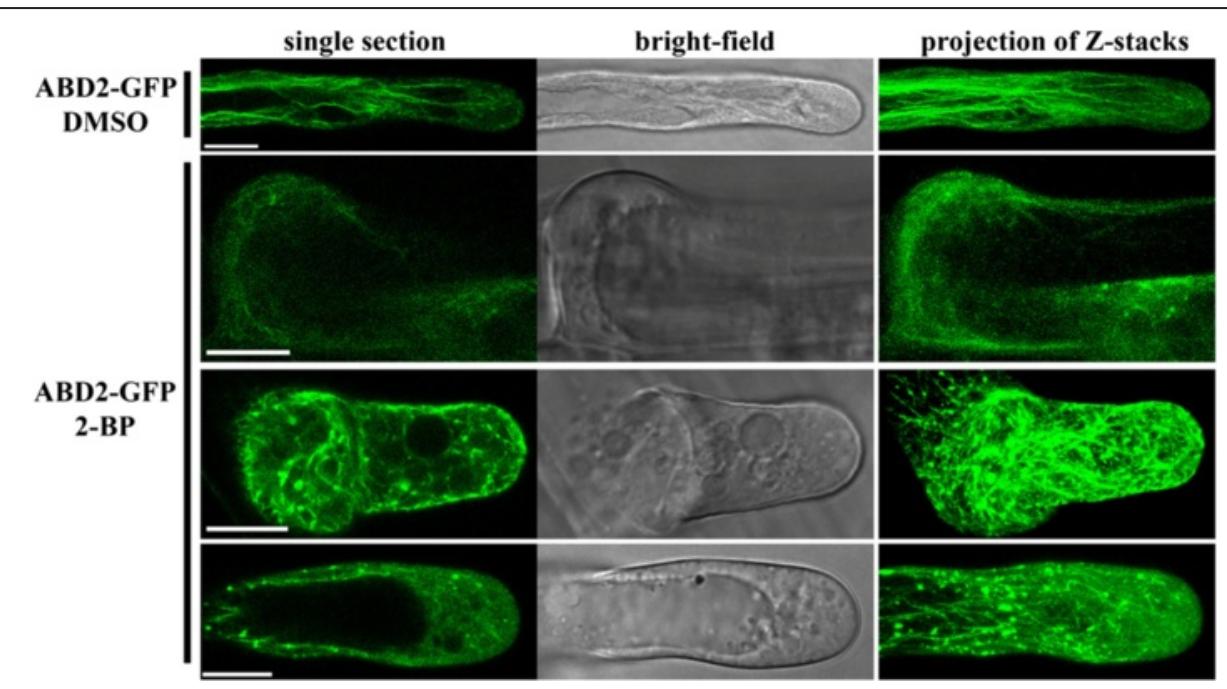

Figure 4 2-BP induced fragmentation and cross-linking of actin MF in root hairs. 4 DAG seedlings of Pro 35 : GFP-ABD2-GFP transgenic seedlings were treated with DMSO or with $10 \mu \mathrm{M}$ 2-BP for 2-3 hr before imaging. 18 to 20 root hairs at different stages were examined and representative images are shown. Single section indicates one optical section taken at the mid-plane of a root hair. For each root hair shown, twenty $1 \mu \mathrm{m}$ optical sections were superimposed to generate the projection of Z-stacks. Bars $=10 \mu \mathrm{m}$. 
sensor line [41] with 2-BP or with DMSO. The P15Y sensor line expresses a Pro $_{U B Q 10}$-driven $\mathrm{PIP}_{2}$-binding TUBBY-C fused with CITRINE [41]. As shown by other $\mathrm{PIP}_{2}$ sensors [42], $\mathrm{PIP}_{2}$ was asymmetrically localized at the PM of initiation sites in trichoblasts (Figure 5A). During hair elongation, $\mathrm{PIP}_{2}$ maintained its asymmetric $\mathrm{PM}$ localization at the apical region (Figure 5B). Application of 2-BP significantly redistributed fluorescence signals from the PM to cytosol (Figure 5C,D,E), suggesting abolished $\mathrm{PIP}_{2}$ at the PM. For root hairs either at the initiation stage (Figure $5 \mathrm{C}$ ) or at the elongating stage (Figure 5D), PIP 2 was detected mostly in the cytoplasm and hardly at all at the PM. The residual signals at the $\mathrm{PM}$ were uniform (Figure 5D) rather than asymmetric (Figure 5B).

The effect of 2-BP on the dynamic polymerization of actin $\mathrm{MF}$ and $\mathrm{PIP}_{2}$ distribution indicated polarity defects. Because actin MF and $\mathrm{PIP}_{2}$ distribution in tip-growing plant cells are regulated by or associated with ROP GTPases $[43,44]$ that are subjected to palmitoylation [8-10], we wondered if 2-BP treatment could redistribute ROP GTPases into the cytoplasm or cause an uniform localization at the PM rather than the apex-restricted PM localization in root hairs $[43,45]$. To this end, we applied either DMSO or 2-BP to Pro F :GFP-ROP2 transgenic seedlings in which ROP2, the key ROP GTPase regulating root hair growth [43], was driven by a root hair-specific promoter [46]. ROP2 was concentrated at the PM of hair initiation sites in trichoblasts of $\operatorname{Pro}_{E 7}$ : GFP-ROP2 transgenic seedlings treated with DMSO (Figure 6A), as previously reported [43,45,47]. Expression of ROP2 caused root hair bulging (Figure 6A,B,C). Likely due to the overexpression effect, ectopic ROP2 signals were detected along the PM as well as in the cortical cytoplasm (Figure 6A,B,C). By contrast, 2-BP treatment induced rapid re-localization of ROP2 from the PM to the cytoplasm (Figure 6D,E,F). Significant differences were observed as early as 30 min after 2-BP treatment (Figure 6G).

\section{RabA4b-positive post-Golgi secretion was impaired by 2-BP in root hairs}

Polarized growth requires regulated exocytosis to deliver building materials for membranes and cell walls. In Arabidopsis root hairs, RabA4b-mediated secretory vesicles form an inverted cone-shaped pattern critical for polarized growth $[26,27,29,30]$. To determine the effect of 2-BP on RabA4b-positive post-Golgi secretion, we applied either 2-BP or DMSO to 4 DAG seedlings transformed with

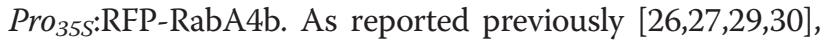
RFP-RabA4b was dynamically distributed to the apical cytoplasm in the form of an inverted cone with a trail in growing root hairs, which was not disturbed by DMSO (Figure 7A). Such a distribution pattern was dynamically
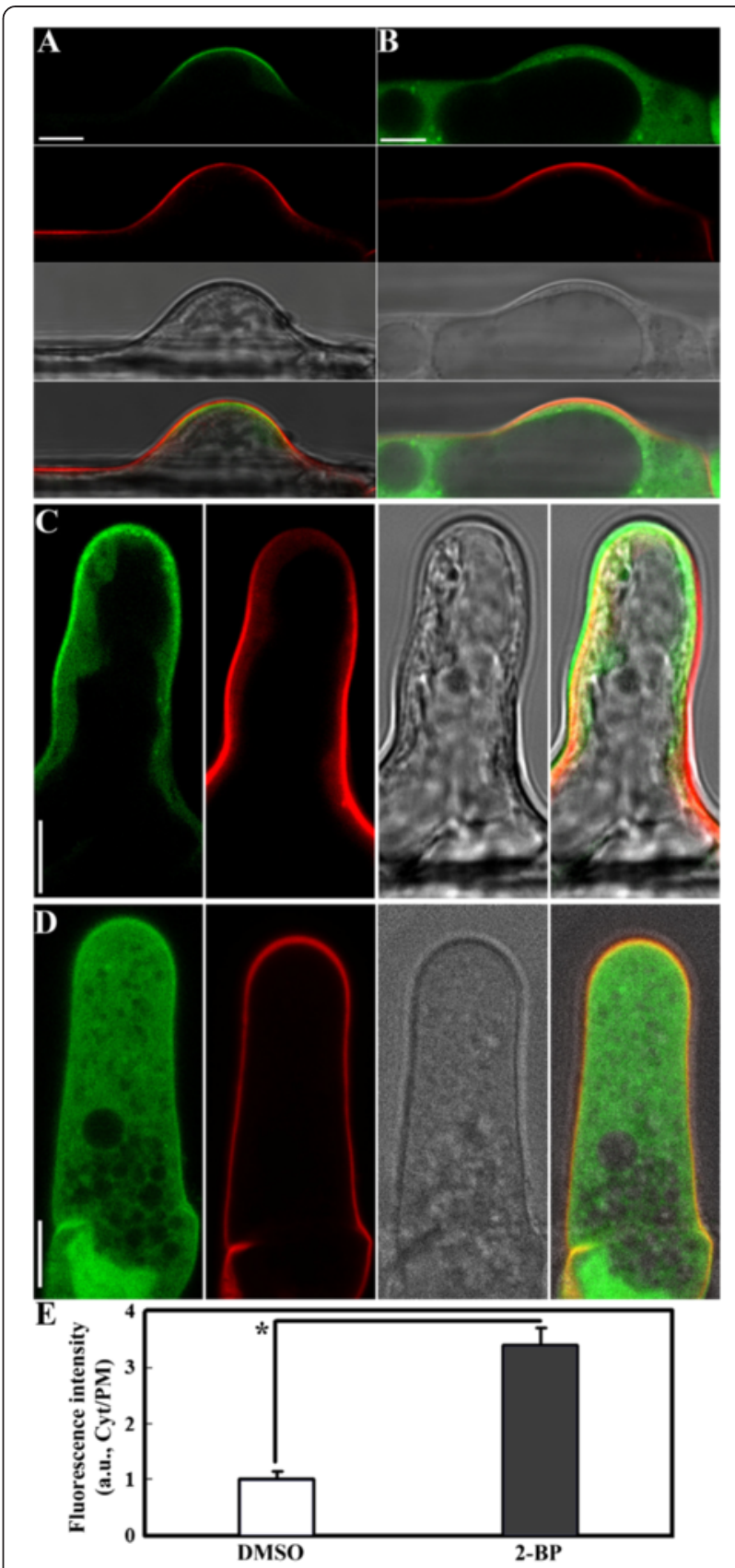

Figure 5 2-BP treatment re-distributed the $\mathrm{PIP}_{2}$ sensor from the PM to the cytoplasm in root hairs. A. DMSO-treated root hairs expressing the $\mathrm{PIP}_{2}$ sensor (green) at the initiating stage. B. 2-BP-treated root hairs expressing the $\mathrm{PIP}_{2}$ sensor at the initiating stage. $\mathbf{C}$. DMSOtreated root hairs expressing the $\mathrm{PIP}_{2}$ sensor at the elongating stage. D. 2-BP-treated root hairs expressing the $\mathrm{PIP}_{2}$ sensor at the elongating stage. E. Ratio of fluorescence signals. a.u. stands for arbitrary fluorescence units. Cyt/PM indicates the ratio of cytoplasmic to the plasma membrane signal. Results are means \pm standard deviation $(S D, n=30)$. Asterisk indicates significant difference (Student's $t$-test, $P<0.01$ ). Root hairs were stained with the fluorescence dye propidium iodide (red) to outline cell shape. Corresponding bright-field images are shown together with merges of different channels. Bars $=7.5 \mu \mathrm{m}$. 


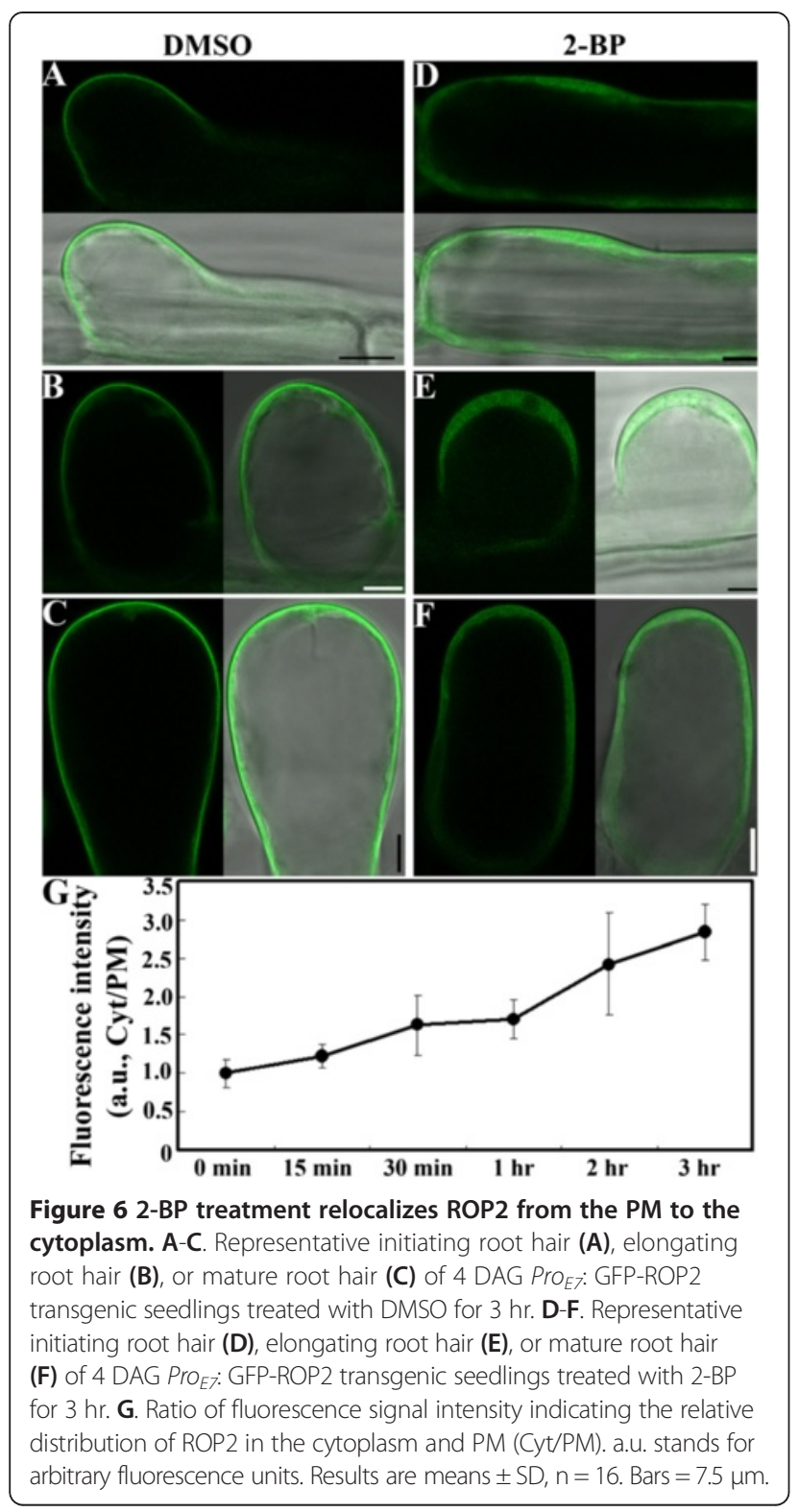

maintained as long as root hairs grew (Figure 7E, Additional file 2: Movie S1). Application of 2-BP incurred two noticeable effects in root hairs: it disrupted the tipfocused inverted cone pattern and caused aggregation of RabA4b-positive vesicles (Figure 7B-D,F, Additional file 3: Movie S2). The effects of 2-BP were observed as early as $2 \mathrm{hr}$ after treatment (Figure 7B-D), suggesting that postGolgi secretory trafficking was sensitive to the inhibition of protein palmitoylation. Disruption of the tip-focused RabA4b-distribution pattern correlated with the growth kinetics of root hairs, in that RabA4b was more concentrated in the apical region than in the shank region in growing root hairs (Figure 7C) whereas completely dissipated into punctates in non-growing root hairs
(Figure 7D). Despite the disruption on RabA4b-positive secretory trafficking, by following single aggregates during time-lapse confocal fluorescence microscopy, we observed that at least some vesicles were able to be exocytosed (Figure 7F).

\section{2-BP inhibits endocytic and vacuolar trafficking}

The disrupted RabA4b distribution pattern by 2-BP prompted us to test whether endocytosis was affected because polarized growth requires balanced exocytosis and endocytosis to maintain the dynamic integrity of the cell membranes and walls. FM4-64 enters into plant cells through the PM by endocytosis and eventually reaches the tonoplast [33]. To determine whether 2-BP interfered with endocytic trafficking, we pre-treated 4 DAG seedlings with $10 \mu \mathrm{M} 2$-BP or DMSO for 2 hrs before pulse-labeling the roots with $4 \mu \mathrm{M}$ FM4-64. In both initiating (Figure 8A) and elongating root hairs (Figure 8C) pre-treated with DMSO, FM4-64 was internalized from the PM into the TGN/EE (Additional file 4: Movie S3). By contrast, no cytosolic vesicles were observed in root hairs pre-treated with 2-BP during the time course of the experiment (Additional file 5: Movie S4) either in initiating root hairs (Figure 8B) or in elongated root hairs (Figure 8D), indicating complete inhibition of endocytosis.

Endocytic trafficking starts at the PM and ends at the vacuole. To find out whether vacuolar trafficking was influenced by 2-BP treatment, we followed the endocytic trafficking of FM4-64 to the tonoplast. FM4-64 labeled both cytosolic vesicles and the tonoplast after 3040 min uptake in root hairs (Figure 9A). Because BFA treatment caused aggregation of FM4-64-labeled TGN/ EE into BFA compartments (Figure 9B), we reasoned that a BFA washout would allow us to examine the process of vacuolar trafficking from TGN/EE via PVC/ MVB to vacuoles. In root hairs treated with DMSO, BFA washout led to the labeling of FM4-64 of the tonoplast (Figure 9C), indicating undisturbed trafficking from the TGN/EE to vacuoles. However, in the presence of 2-BP, BFA washout resulted in dissipation of FM4-64 signals from the BFA compartments (Figure 9D). Furthermore, FM4-64 was redistributed mostly to the PM rather than to the tonoplast (Figure 9D), suggesting that 2-BP caused mis-sorting of vesicles originally destined to vacuoles.

To further support the idea that 2-BP inhibited vacuolar trafficking, we applied either 2-BP or DMSO to root hairs expressing YFP-2XFYVE, which binds specifically to PI3P [41]. Because PI3P goes to vacuoles for degradation through vacuolar trafficking routes from TGN/EE to $\mathrm{PVC} / \mathrm{MVB}$, we reasoned that this would serve as a good biosensor for vacuolar trafficking [48]. Indeed, 2$\mathrm{BP}$ but not DMSO induced the formation of ring-shaped compartments positive for PI3P, to an extent similar to 

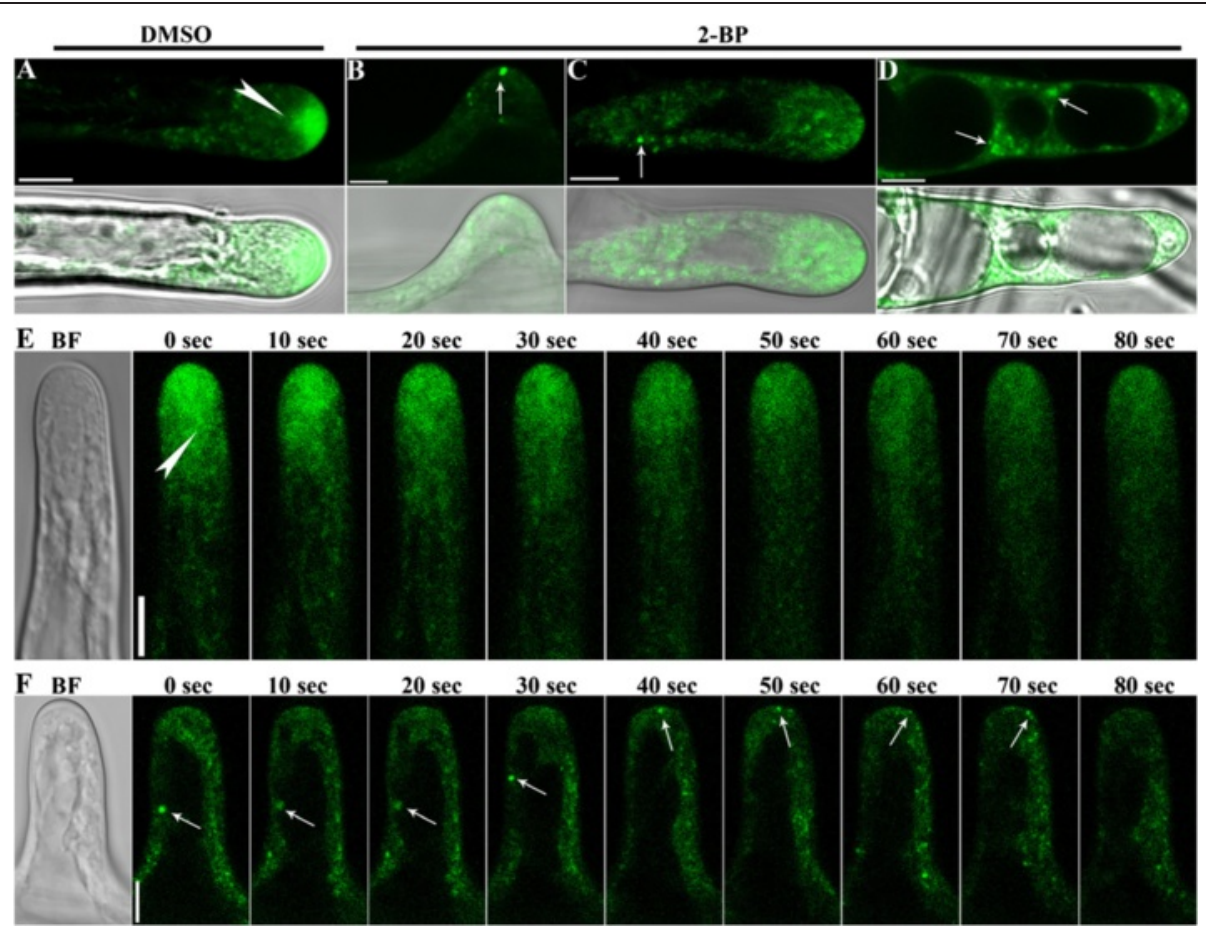

Figure 7 2-BP treatment interfered with the dynamics of RabA4b-positive secretory vesicles. A. Distribution of RabA4b-positive vesicles in a growing root hair of 4 DAG RFP-RabA4b transgenic seedlings treated with DMSO for $2 \mathrm{hr}$. The arrowhead points at the base of the clear zone where RabA4b-positive vesicles form an inverted cone. Below is merge of fluorescence and bright field images. B-D. Distribution of RabA4bpositive vesicles in a root hair right after initiation (B), a growing root hair (C), or an arrested root hair (D) of 4 DAG RFP-RabA4b transgenic seedlings treated with $10 \mu \mathrm{M}$ 2-BP for $2 \mathrm{hr}$. The arrows point at enlarged vesicles positive for RabA4b. Below are the merges of fluorescence and bright field images. E-F. RFP-RabA4b fluorescence was visualized in root hairs treated with DMSO (E) or $10 \mu \mathrm{M}$ 2-BP (F) for 2 hr using time-lapse confocal fluorescence microscopy. Left-most are the bright-field images. The arrowhead points to the base of the clear-zone. The arrows follow the moving track of a single large vesicle over time. Bars $=7.5 \mu \mathrm{m}$.

but less substantial than that caused by wortmannin (Additional file 1: Figure S6), through which PVC/MVBs on their way to vacuoles fuse to form ring-shaped compartments [49]. These results indicated that vacuole trafficking through the TGN/EE and PVC/MVBs was compromised by 2 -BP.

\section{Discussion}

As a reversible post-translational modification, protein palmitoylation has been extensively studied in polarized cell growth in metazoans [6]. By using pharmacological and genetic approaches, we demonstrate that protein palmitoylation, contributed primarily by the Golgi-localized TIP1 (Figure 3, Additional file 1: Figure S4) and secondarily by PATs from other endomembrane compartments such as vacuoles (Figure 3), plays a key role in the polar growth of root hairs. By using the tonoplast-cytoplasm partition of CBL2 as an indicator for effective inhibition of palmitoylation, we determined the application regime of 2-BP on root hair growth (Figure 1). 2-BP has been used extensively in yeast and metazoans $[1,31]$ but rarely in plants $[11,12,18]$. Treatment with 2-BP resulted in shorter and wider root hairs (Figure 2), suggesting compromised hair elongation and polarity due to inhibited palmitoylation. Functional loss of PAT10 resulted in shorter root hairs but affected width (Figure 2) indicate that PAT10 functions in hair elongation but not in polarity control. Treatment of 2-BP resulted in an additional reduction in hair length in pat10-2 (Figure 2), suggesting that other PATs are involved in hair elongation. In contrast to pat10-2, in tip1-4 neither hair length or width was significantly affected by 2-BP (Figure 2), suggesting that TIP1 is the primary PATs controlling root hair elongation and polarity. However, 2-BP does induce an expansion of root hair initiation zone in tip1-4 as in wild type (Figure 2), indicating that other PATs also contribute to hair elongation, at least in specific context. Indeed, multiple PATs are expressed in root hairs (Additional file 1: Figure S2) besides TIP1 and PAT10 and their diverse subcellular distributions as revealed by a transient expression assay [17] hinted at a complex effect of protein palmitoylation on root hair growth.

Root hair growth requires dynamic distribution of polarity proteins, among which ROP GTPases [43,45-47] are crucial. As their yeast and metazoan counterparts [6,7], ROP GTPases are palmitoylated proteins whose membrane distribution and activities rely on their 


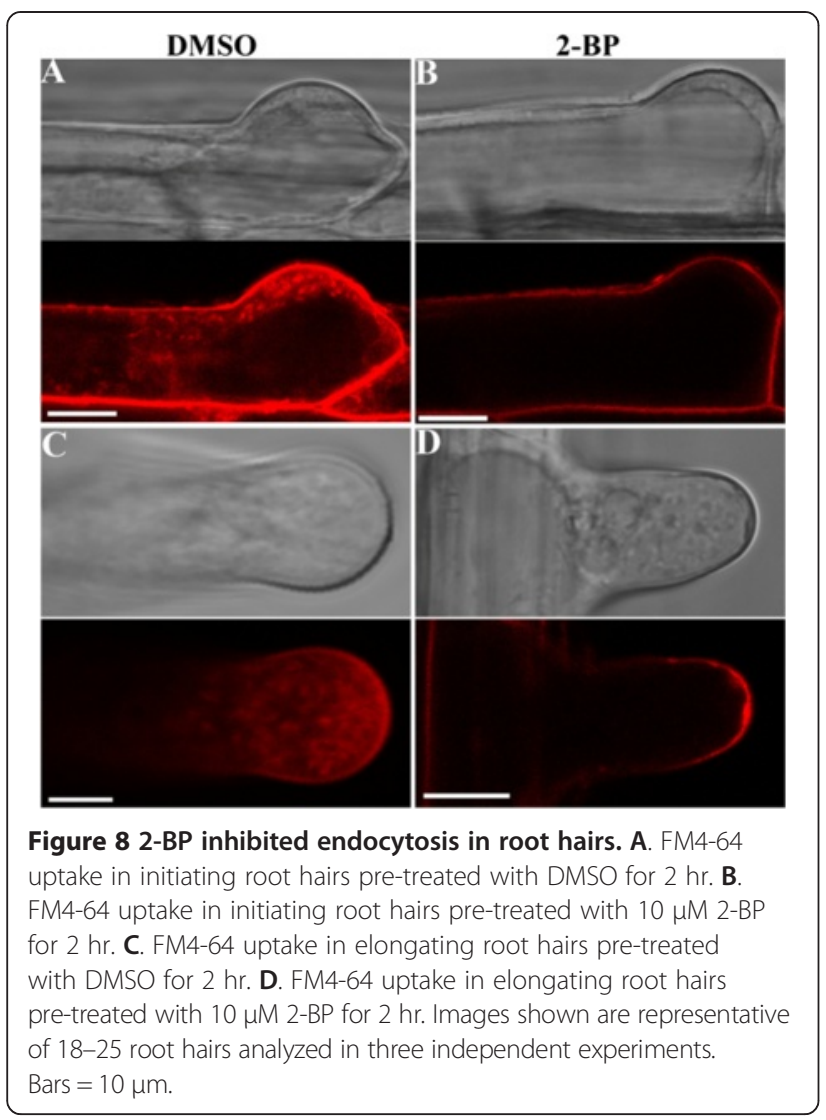

palmitoylation status $[9,10]$. We showed that 2-BP causes a significant translocation of ROP2 from the PM to the cytoplasm (Figure 6), indicating membrane dissociation due to reduced palmitoylation. However, palmitoylation of several ROP GTPases was shown to be crucial for their partitioning among membrane microdomains rather than between the PM and the cytoplasm $[9,10]$. The discrepancy could be due to the specific property of tip-growing root hair cells, in which heterogeneity of the PM is spatially reflected on a much larger scale than in microdomains of non-polar growing cells [50].

As central regulators of polarized cell growth in plants [50,51], ROP GTPases play critical roles in multiple intracellular activities, most importantly, the dynamic polymerization of actin MF [43] that is crucial for maintaining polar growth in root hairs [21-24]. Treatment of 2-BP cause substantial fragmentation of actin MF (Figure 4), indicating impaired actin MF polymerization. However, the effect of 2-BP is different from that induced by the actin MF depolymerization drug LatB (Additional file 1: Figure S4) such that 2-BP results in extensive cross-linking as indicated by strong puncta at the interaction of several short actin bundles (Figure 4). The dissociation of ROP GTPases from the PM of root hairs (Figure 6) only partially explains the effect because interfering with ROP activities in root hairs by expressing

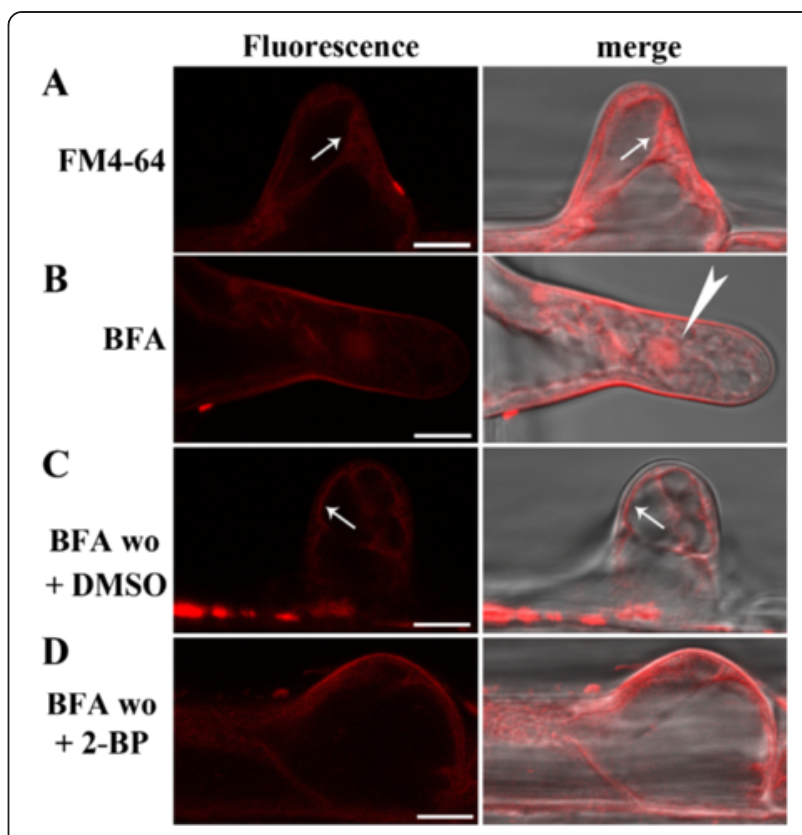

Figure 9 2-BP interfered with vacuolar trafficking. A-D. 4 DAG WT seedlings were pulse-labeled with FM4-64, washed and incubated for $30 \mathrm{~min}$ (A), or pulse-labeled with FM4-64 followed by $30 \mathrm{~min}$ incubation with 1/2 MS medium supplemented with $50 \mu \mathrm{M}$ BFA (B). BFA-treated seedlings were then washed with $1 / 2$ MS medium supplemented with either DMSO (BFA wo + DMSO) (C) or 2-BP (BFA wo + 2-BP) (D). Arrows point at the tonoplat labeled by FM4-64. Arrowhead indicates the BFA compartment. Results are representative of 20 root hairs for each treatment. Bars $=7.5 \mu \mathrm{m}$.

a dominant negative ROP2 [43] does not result in the disorganized actin MF network. A more likely scenario is that other palmitoylated proteins than ROPs may regulate actin MF dynamics in root hairs, as was reported for some receptor kinases during neuronal growth [6].

Polar growth of root hairs requires restricted delivery of secretory vesicles $[28,52]$. RabA4s are critical for postGolgi secretion in root hairs $[26,27,29,30]$ by forming an inverted cone-shaped vesicle stream to deliver materials for growth. We showed that 2-BP dissipates the tipfocused distribution pattern of RabA4b-positive postGolgi secretory vesicles and caused their aggregation (Figure 7). Because post-Golgi vesicles rely on dynamic polymerization of actin MF for their motility and possibly for their directionality in root hairs [24,28], the disrupted actin MF network due to 2-BP (Figure 4) may have indirectly resulted in the impaired secretion (Figure 7).

Endocytosis not only retrieves excess materials delivered from exocytosis to maintain cellular homeostasis but also mediates the membrane distribution of key signaling proteins during polar growth [50]. As a key signaling molecule, $\mathrm{PIP}_{2}$ was recently shown to regulate clathrin-mediated endocytosis [53]. By using a fluorescence probe specific for $\mathrm{PIP}_{2}$ [41], we showed that 2-BP causes a rapid loss of $\mathrm{PIP}_{2}$ at the PM (Figure 5), which 
correlates with the complete inhibition of endocytosis by 2-BP pre-treatment (Figure 8). In addition to internalization from the PM, vacuolar trafficking from the TGN/EE is also compromised by 2-BP (Figure 9, Additional file 1: Figure S6). Rather than proceeding to the tonoplast from the TGN/EE, FM4-64 instead traffics to the PM (Figure 9), indicating defective vacuolar trafficking. By using a fluorescence probe specific for PI3P, we showed that 2-BP caused homotypic fusion of PVC/MVBs rather than fusion of PVC/MVBs to vacuoles (Additional file 1: Figure S6). The dramatic responses of membrane trafficking to 2-BP suggests that key proteins regulating membrane trafficking in plant cells are controlled by protein palmitoylation. SNAREs are critical components in vesicle trafficking machinery critical for selective vesicle fusion [48]. Many SNAREs are modified by palmitoylation in yeast and metazoans and such palmitoylation might be evolutionarily conserved $[2,4]$. The Arabidopsis genome encodes a large number of SNAREs [48] that are localized differentially at Golgi and postGolgi compartments [54]. Combining genetic analyses and dynamic subcellular targeting of these SNAREs may reveal important substrates of protein palmitoylation during root hair growth.

\section{Conclusions}

As a reversible post-translational modification that often regulates subcellular targeting and activities of signaling proteins, protein palmitoylation has been demonstrated to be critical for polar growth in metazoans. By using genetic as well as pharmacological approaches, we show here that protein palmitoylation, regulated by protein $\mathrm{S}$-acyl transferases from different endomembrane compartments such as Golgi and vacuole, is critical for the polar growth of root hairs in Arabidopsis. Inhibition of protein palmitoylation by application of 2-BP disturbed key intracellular activities in root hairs, including actin MF polymerization, the asymmetric distribution of $\mathrm{PIP}_{2}$, post-Golgi secretion, as well as endocytic trafficking. Although some of the effects were likely indirect, the cytological data reported here will contribute to a deep understanding of protein palmitoylation during tip growth in plants.

\section{Methods}

\section{Plant materials and growth conditions}

The T-DNA insertion line, SALK_089971C (tip1-4), was obtained from the Arabidopsis Biological Resource Center (ABRC, http://www.arabidopsis.org). Primers F1/R1 were used to characterize TIP1 expression in tip1-4. Arabidopsis thaliana Col-0 ecotype was used as wild type. Arabidopsis plants were grown as described [12]. For seedlings growing on plates, surface-sterilized Arabidopsis seeds were grown on Murashige and Skoog basal medium with vitamins (MS) (Phytotechlab, http://www. phytotechlab.com/) except where noted. Plates were kept at $4^{\circ} \mathrm{C}$ in darkness for 4 days before being transferred to a growth chamber with a 16-h light:8-h dark cycle at $21^{\circ} \mathrm{C}$. Transgenic plants were selected on MS medium supplemented with $30 \mu \mathrm{g} / \mathrm{ml}$ Basta salt (Sigma, http:// www.sigmaaldrich.com/).

\section{Plasmid construction}

All vectors were generated using the Gateway ${ }^{\text {ma }}$ technology (Invitrogen). Entry vectors for the coding sequence of $C B L 2$ and the whole genomic sequence of TIP1 including its native promoter were generated in the pENTRY/SD/D-TOPO (Invitrogen) using the primer pair ZP595/ZP596 for CBL2 and ZP533/ZP534 for TIP1g. The destination vector Pro $_{U B Q 10}: G W-R F P$ was generated by replacing the $\operatorname{Pro}_{355}$ promoter with Pro $_{U B Q 10}$ using the primer pair ZP510/ZP511 with the SpeI/HindIII double digestion sites from a previously described destination vector [55]. TIP1g-GFP was generated by an LR reaction using a GW:GFP translation fusion destination vector [12] and the TIP1g entry vector. Pro $_{E 7}$ :GFP-ROP2 was generated by a LR reaction using the Pro $_{E 7}: \mathrm{GFP}-\mathrm{GW}$ destination vector and the entry vector for ROP2 [46]. All PCR amplifications used Phusion ${ }^{\mathrm{TM}}$ hot start high-fidelity DNA polymerase with the annealing temperature and extension times recommended by the manufacturer (Finnzyme). All entry vectors were sequenced and verified. The Bioneer PCR purification kit and the Bioneer Spin miniprep kit were used for PCR product recovery and plasmid DNA extraction, respectively. Primers are listed in (Additional file 1: Table S1).

\section{Quantification of root hair length and width}

In the presence of 2-BP or DMSO, the region of root growth and root hair expansion was $1-1.5 \mathrm{~mm}$ distal from the primary root tip of 4 DAG seedlings and was thus chosen for length and width measurements. Images of that region were taken from individual seedlings using an Axio Observer A1 equipped with a CCD camera (Zeiss). Quantification of root hair length and width was performed according to previous descriptions [46] using ImageJ (http://rsbweb.nih.gov/ij/).

\section{Pharmacological treatments}

Stock solutions of various inhibitors (Sigma) were prepared using DMSO as the solvent at the following concentrations: $50 \mathrm{mM}$ 2-BP, $35 \mathrm{mM}$ BFA, $20 \mathrm{mM}$ CHX and $4 \mathrm{mM}$ FM4-64. Stock solutions were diluted and added to $1 / 2 \mathrm{MS}$ at designated final concentrations, i.e. 10-50 $\mu \mathrm{M}$ 2-BP, $50 \mu \mathrm{M}$ BFA, $50 \mu \mathrm{M}$ CHX, and $4 \mu \mathrm{M}$ FM4-64. DMSO was similarly diluted for the controls. All experiments were repeated at least three times. 
Images and movies shown are representative of approximately 18-30 root hairs.

\section{Fluorescence labeling, quantification, and microscopy}

Fluorescent images were captured using a Leica TCS SP5II confocal laser scanning microscope (Leica, Wetzlar, Germany) with a Plan-Neofluar $40 \times / 1.3 \mathrm{NA}$ oil DIC objective or $63 \times / 1.45 \mathrm{NA}$ oil DIC objectives. GFPRFP double-labeled plant materials were captured alternately using line switching with the multi-track function (488 $\mathrm{nm}$ for GFP and $545 \mathrm{~nm}$ for RFP). Fluorescence was detected using a 505- to 550-nm band-pass filter for GFP and a 575- to 650-nm band-pass filter for RFP. YFP-RFP double-labeled plant materials were captured alternately using line switching with the multi-track function (514 nm for YFP and $545 \mathrm{~nm}$ for RFP). Fluorescence was detected using a 530- to 580-nm band-pass filter for YFP and a 575- to 650-nm band-pass filter for RFP. Post-acquisition image processing was performed with the LAS AF Lite image processing software (Leica). For quantification of $\mathrm{PIP}_{2-(\mathrm{Cyt} / \mathrm{PM})}, \mathrm{ROP}_{-(\mathrm{Cyt} / \mathrm{PM})}$, and

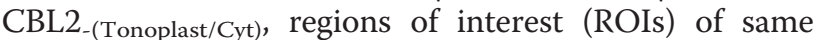
sizes were designated at the PM, the cytoplasm or the tonoplast. Average signal intensity at ROIs was measured using ImageJ and ratio of the cytoplasm to the PM (for $\mathrm{PIP}_{2}$ ), the PM to the cytoplasm (for ROP2) or the tonoplast to the cytoplasm (for CBL2) was calculated. All vacuolated areas were excluded from the cytoplasm ROI.

\section{Accession numbers}

Arabidopsis Genome Initiative locus identifiers for the genes mentioned in this article are: At2g40990 for PAT2; At5g05070 for PAT3; At3g56930 for PAT4; At3g48760 for PAT5; At5g41060 for PAT6; At3g26935 for PAT7; At4g24630 for PAT8; At5g50020 for PAT9; At3g51390 for PAT10; At4g22750 for PAT13; At3g60800 for PAT14; At5g04270 for PAT15; At3g09320 for PAT16; At3g04970 for PAT17; At4g01730 for PAT18; At3g22180 for PAT20; At2g33640 for PAT21; At1g69420 for PAT22; At5g20350 for TIP1/PAT24; At5g55990 for CBL2; At1g12560 for EXP7; At1g20090 for ROP2; and At4g39990 for RabA4b.

\section{Additional files}

Additional file 1: Six supplemental figures and their corresponding legends.

Additional file 2: A movie showing dynamic distribution of RFP-RabA4b in a root hair treated with DMSO for $2 \mathrm{hr}$.

Additional file 3: A movie showing dynamic distribution of RFP-RabA4b in a root hair treated with $10 \mu \mathrm{M}$ 2-BP for $2 \mathrm{hr}$.

Additional file 4: A movie showing uptake of FM4-64 in root hairs pre-treated with DMSO for $2 \mathrm{hr}$.
Additional file 5: A movie showing uptake of FM4-64 in root hairs pre-treated with $10 \mu \mathrm{M}$ 2-BP for $2 \mathrm{hr}$.

\section{Abbreviations}

2-BP: 2-bromopalmitate; BFA: Brefeldin A; CBL: Calcineurin B like proteins; DAG: Days after germination; DMSO: Dimethyl sulfoxide; ER: Endoplasmic reticulum; LatB: Latrunculin B; MF: Microfilaments; PAT: Protein S-acyl transferases; PIP : Phosphatidylinositol $(4,5)$-bisphosphate; PM: Plasma membrane; PVC/MVB: Prevacuolar compartment/multivesicular bodies; SNAREs: N-ethylmaleimide-sensitive factor-activating protein receptors: TIP1: TIP GROWTH DEFECTIVE1; TGN/EE: trans-Golgi network/early endosomes; TM: Transmembrane.

\section{Competing interests}

The authors declare that they have no competing interests.

\section{Authors' contributions}

Y.-L. Z., E. L., Q.-N. F., X.-Y. Z., F.-R. G., performed the research and analyzed the data. S. L. designed the research and analyzed the data. S. L. and Y. Z. wrote the manuscript. All authors read and approved the final manuscript

\section{Acknowledgements}

We thank Dr. Sheila McCormick for critical reading and language editing of this manuscript, Dr. Yvon Jaillais for the PIP 2 and PI3P sensor lines, Dr. Elison B. Blancaflor for the Pro 355 : GFP-ABD2-GFP marker line, and ABRC for the tip 1-4 mutant. We thank Dr. Xian Sheng Zhang for giving us access to the microscope facilities of his laboratory. This work was supported by Shandong Provincial Natural Science Foundation, China (ZR2014CM027 to S. L.) and by a grant from the National Science Foundation of China (NSFC)

(31261160490). Y. Z.'s laboratory is supported by the Tai-Shan Scholar program from Shandong Provincial Government.

Received: 8 January 2015 Accepted: 23 January 2015

Published online: 13 February 2015

\section{References}

1. Greaves J, Chamberlain LH. DHHC palmitoyl transferases: substrate interactions and (patho) physiology. Trends Biochem Sci. 2011;36(5):245-53.

2. Hemsley PA, Grierson CS. Multiple roles for protein palmitoylation in plants. Trends Plant Sci. 2008;13(6):295-302.

3. Magee T, Seabra MC. Fatty acylation and prenylation of proteins: what's hot in fat. Curr Opin Cell Biol. 2005;17(2):190-6.

4. Running MP. The role of lipid post-translational modification in plant developmental processes. Front Plant Sci. 2014;5:50

5. Hemsley PA, Weimar T, Lilley KS, Dupree P, Grierson CS. A proteomic approach identifies many novel palmitoylated proteins in Arabidopsis. New Phytol. 2013;197(3):805-14.

6. Kang R, Wan J, Arstikaitis P, Takahashi H, Huang K, Bailey AO, et al. Neura palmitoyl-proteomics reveals dynamic synaptic palmitoylation. Nature. 2008:456(7224):904-9.

7. Roth AF, Wan J, Bailey AO, Sun B, Kuchar JA, Green WN, et al. Global analysis of protein palmitoylation in yeast. Cell. 2006:125(5):1003-13.

8. Sorek N, Henis Yl, Yalovsky S. How prenylation and S-acylation regulate subcellular targeting and function of ROP GTPases. Plant Signal Behav. 2011;6(7):1026-9.

9. Sorek N, Poraty L, Sternberg H, Bar E, Lewinsohn E, Yalovsky S. Activation status-coupled transient $S$ acylation determines membrane partitioning of a plant Rho-related GTPase. Mol Cell Biol. 2007;27(6):2144-54.

10. Sorek N, Segev O, Gutman O, Bar E, Richter S, Poraty L, et al. An S-acylation switch of conserved $\mathrm{G}$ domain cysteines is required for polarity signaling by ROP GTPases. Curr Biol. 2010;20(10):914-20.

11. Batistic $O$, Rehers M, Akerman A, Schlucking $K$, Steinhorst L, Yalovsky S, et al. S-acylation-dependent association of the calcium sensor CBL2 with the vacuolar membrane is essential for proper abscisic acid responses. Cell Res. 2012:22(7):1155-68

12. Zhou L-Z, Li S, Feng Q-N, Zhang Y-L, Zhao X, Zeng Y-I, et al. PROTEIN $S-A C Y L$ TRANSFERASE10 is critical for development and salt tolerance in Arabidopsis. Plant Cell. 2013;25(3):1093-107. 
13. Adjobo-Hermans MJ, Goedhart J, Gadella Jr TW. Plant G protein heterotrimers require dual lipidation motifs of $\mathrm{Ga}$ and $\mathrm{Gr}$ and do not dissociate upon activation. J Cell Sci. 2006;119(Pt 24):5087-97.

14. Zeng Q, Wang X, Running MP. Dual lipid modification of Arabidopsis $\mathrm{G}$-subunits is required for efficient plasma membrane targeting. Plant Physiol. 2007;143(3):1119-31.

15. Gagne JM, Clark SE. The Arabidopsis stem cell factor POLTERGEIST is membrane localized and phospholipid stimulated. Plant Cell. 2010;22 (3):729-43.

16. Ohno Y, Kihara A, Sano T, Igarashi Y. Intracellular localization and tissue-specific distribution of human and yeast DHHC cysteine-rich domain-containing proteins. Biochim Biophys Acta. 2006;1761(4):474-83.

17. Batistic O. Genomics and localization of the Arabidopsis DHHC-CRD Sacyltransferase protein family. Plant Physiol. 2012. doi:10.1104/pp.1112.203968.

18. Hemsley PA, Kemp AC, Grierson CS. The TIP GROWTH DEFECTIVE1S-acyl transferase regulates plant cell growth in Arabidopsis. Plant Cell. 2005;17 (9):2554-63.

19. Ryan E, Grierson CS, Cavell A, Steer M, Dolan L. TIP1 is required for both tip growth and non-tip growth in Arabidopsis. New Phytol. 1998;138(1):49-58.

20. Schiefelbein J, Galway M, Masucci J, Ford S. Pollen tube and root-hair tip growth is disrupted in a mutant of Arabidopsis thaliana. Plant Physiol. 1993;103(3):979-85.

21. Baluska F, Salaj J, Mathur J, Braun M, Jasper F, Samaj J, et al. Root hair formation: F-actin-dependent tip growth is initiated by local assembly of profilin-supported F-actin meshworks accumulated within expansinenriched bulges. Dev Biol. 2000;227(2):618-32.

22. Ringli C, Baumberger N, Diet A, Frey B, Keller B. ACTIN2 is essential for bulge site selection and tip growth during root hair development of Arabidopsis. Plant Physiol. 2002;129(4):1464-72.

23. Ringli C, Baumberger N, Keller B. The Arabidopsis root hair mutants der2-der9 are affected at different stages of root hair development. Plant Cell Physiol. 2005:46(7):1046-53.

24. Voigt B, Timmers ACJ, Šamaj J, Hlavacka A, Ueda T, Preuss M, et al. Actin-based motility of endosomes is linked to the polar tip growth of root hairs. Euro J Cell Biol. 2005;84(6):609-21.

25. Ovecka M, Lang I, Baluska F, Ismail A, Illes P, Lichtscheidl IK. Endocytosis and vesicle trafficking during tip growth of root hairs. Protoplasma. 2005;226(1-2):39-54

26. Preuss ML, Schmitz AJ, Thole JM, Bonner HK, Otegui MS, Nielsen E. A role for the RabA4b effector protein PI-4KB1 in polarized expansion of root hair cells in Arabidopsis thaliana. J Cell Biol. 2006;172(7):991-8.

27. Preuss ML, Serna J, Falbel TG, Bednarek SY, Nielsen E. The Arabidopsis Rab GTPase RabA4b localizes to the tips of growing root hair cells. Plant Cell. 2004;16(6):1589-603.

28. Samaj J, Muller J, Beck M, Bohm N, Menzel D. Vesicular trafficking, cytoskeleton and signalling in root hairs and pollen tubes. Trends Plant Sci. 2006;11(12):594-600.

29. Yoo CM, Blancaflor EB. Overlapping and divergent signaling pathways for ARK1 and AGD1 in the control of root hair polarity in Arabidopsis thaliana. Front Plant Sci. 2013;4:528.

30. Yoo CM, Quan L, Cannon AE, Wen J, Blancaflor EB. AGD1, a class 1 ARF-GAP acts in common signaling pathways with phosphoinositide metabolism and the actin cytoskeleton in controlling Arabidopsis root hair polarity. Plant J. 2012;69(6):1064-76.

31. Jennings BC, Nadolski MJ, Ling Y, Baker MB, Harrison ML, Deschenes RJ, et al. 2-Bromopalmitate and 2-(2-hydroxy-5-nitro-benzylidene)-benzo [b] thiophen-3-one inhibit DHHC-mediated palmitoylation in vitro. J Lipid Res. 2009;50(2):233-42.

32. Zimmermann P, Hirsch-Hoffmann M, Hennig L, Gruissem W. GENEVESTIGATOR Arabidopsis microarray database and analysis toolbox Plant Physiol. 2004;136 (1):2621-32.

33. Bolte $\mathrm{S}$, Talbot $\mathrm{C}$, Boutte $\mathrm{Y}$, Catrice $\mathrm{O}$, Read ND, Satiat-Jeunemaitre B. FM-dyes as experimental probes for dissecting vesicle trafficking in living plant cells. J Microsc. 2004;214(Pt 2):159-73.

34. Lam SK, Cai Y, Tse YC, Wang J, Law AH, Pimpl P, et al. BFA-induced compartments from the Golgi apparatus and trans-Golgi network/early endosome are distinct in plant cells. Plant J. 2009;60(5):865-81.

35. Kusano $H$, Testerink $C$, Vermeer JEM, Tsuge $T$, Shimada H, Oka A, et al. The Arabidopsis phosphatidylinositol phosphate 5-kinase PIP5K3 is a key regulator of root hair tip growth. Plant Cell. 2008;20(2):367-80.
36. Thole JM, Vermeer JE, Zhang Y, Gadella Jr TW, Nielsen E. ROOT HAIR DEFECTIVE4 encodes a phosphatidylinositol-4-phosphate phosphatase required for proper root hair development in Arabidopsis thaliana. Plant Cell. 2008:20(2):381-95

37. Vincent $P$, Chua M, Nogue F, Fairbrother A, Mekeel $H$, Xu Y, et al. A Sec14pnodulin domain phosphatidylinositol transfer protein polarizes membrane growth of Arabidopsis thaliana root hairs. J Cell Biol. 2005;168(5):801-12.

38. Wang YS, Motes CM, Mohamalawari DR, Blancaflor EB. Green fluorescent protein fusions to Arabidopsis fimbrin 1 for spatio-temporal imaging of F-actin dynamics in roots. Cell Motil Cytoskeleton. 2004;59(2):79-93.

39. Wang YS, Yoo CM, Blancaflor EB. Improved imaging of actin filaments in transgenic Arabidopsis plants expressing a green fluorescent protein fusion to the $\mathrm{C}$ - and $\mathrm{N}$-termini of the fimbrin actin-binding domain 2. New Phytol. 2008;177(2):525-36.

40. Yoo CM, Wen J, Motes CM, Sparks JA, Blancaflor EB. A class I ADP-ribosylation factor GTPase-activating protein is critical for maintaining directional root hair growth in Arabidopsis. Plant Physiol. 2008;147(4):1659-74.

41. Simon ML, Platre MP, Assil S, van Wijk R, Chen WY, Chory J, et al. A multi-colour/ multi-affinity marker set to visualize phosphoinositide dynamics in Arabidopsis. Plant J. 2014;77(2):322-37.

42. van Leeuwen W, Vermeer JE, Gadella Jr TW, Munnik T. Visualization of phosphatidylinositol 4,5-bisphosphate in the plasma membrane of suspension-cultured tobacco BY-2 cells and whole Arabidopsis seedlings. Plant J. 2007:52(6):1014-26.

43. Jones MA, Shen JJ, Fu Y, Li H, Yang Z, Grierson CS. The Arabidopsis Rop2 GTPase is a positive regulator of both root hair initiation and tip growth. Plant Cell. 2002;14(4):763-76.

44. Kost B, Lemichez E, Spielhofer P, Hong Y, Tolias K, Carpenter C, et al. Rac homologues and compartmentalized phosphatidylinositol 4, 5-bisphosphate act in a common pathway to regulate polar pollen tube growth. J Cell Biol. 1999:145(2):317-30.

45. Molendijk AJ, Bischoff F, Rajendrakumar CS, Friml J, Braun M, Gilroy S, et al. Arabidopsis thaliana Rop GTPases are localized to tips of root hairs and control polar growth. EMBO J. 2001;20(11):2779-88

46. Huang GQ, Li E, Ge FR, Li S, Wang Q, Zhang CQ, et al. Arabidopsis RopGEF4 and RopGEF10 are important for FERONIA-mediated developmental but not environmental regulation of root hair growth. New Phytol. 2013;200 (4):1089-101.

47. Carol RJ, Takeda S, Linstead P, Durrant MC, Kakesova H, Derbyshire P, et al. A RhoGDP dissociation inhibitor spatially regulates growth in root hair cells. Nature. 2005:438(7070):1013-6.

48. Bassham DC, Brandizzi F, Otegui MS, Sanderfoot AA. The secretory system of Arabidopsis. Arabidopsis Book. 2008;6:e0116

49. Wang J, Cai Y, Miao Y, Lam SK, Jiang L. Wortmannin induces homotypic fusion of plant prevacuolar compartments. J Exp Bot. 2009;60(11):3075-83.

50. Bloch D, Yalovsky S. Cell polarity signaling. Curr Opin Plant Biol. 2013;16 (6):734-42.

51. Kost B. Spatial control of Rho (Rac-Rop) signaling in tip-growing plant cells Trends Cell Biol. 2008;18(3):119-27.

52. Gu F, Nielsen E. Targeting and regulation of cell wall synthesis during tip growth in plants. J Integr Plant Biol. 2013;55(9):835-46.

53. Ischebeck T, Werner S, Krishnamoorthy P, Lerche J, Meijon M, Stenzel I, et al. Phosphatidylinositol 4,5-bisphosphate influences PIN polarization by controlling clathrin-mediated membrane trafficking in Arabidopsis. Plant Cell. 2013;25(12):4894-911.

54. Uemura T, Ueda T, Ohniwa RL, Nakano A, Takeyasu K, Sato MH. Systematic analysis of SNARE molecules in Arabidopsis: dissection of the post-Golgi network in plant cells. Cell Struct Funct. 2004;29(2):49-65.

55. Karimi M, Inze D, Depicker A. GATEWAY vectors for Agrobacterium-mediated plant transformation. Trends Plant Sci. 2002;7(5):193-5. 